\title{
Human Body Louse
}

National Cancer Institute

\section{Source}

National Cancer Institute. Human Body Louse. NCI Thesaurus. Code C34906.

Body lice are small, parasitic insects (Pediculus humanus corporis) that feed on human blood, and lay their eggs and deposit their fecal matter on the skin and on clothing. Body lice may be 2 - 3 mm long, as opposed to head and pubic lice which are much smaller. An infestation with body lice causes intense itching, and is transmitted by contact with infested clothing and bedding, as well as by direct contact with a carrier. Risk factors include overcrowded living and/or working conditions and poor hyg iene. 